\title{
Dynamiken subjektiver Erwerbsprekarität in der spåten Erwerbsphase
}

Katrin Golsch und Anja-Kristin Abendroth

\section{Abstract}

Eine Vielzahl an empirischen Studien hat die zunehmende Bedeutung von prekären Beschäftigungsverhältnissen und deren Einfluss auf den individuellen Lebenslauf aufgezeigt. Ein wichtiger Befund dieser Studien ist, dass Prekarität gesellschaftliche Teilhabebedingungen und -chancen einschränkt. Jedoch wird Prekarität in quantitativen Untersuchungen bislang kaum multidimensional und im Längsschnitt betrachtet. Außerdem wird häufig das Augenmerk nur auf die erste Erwerbsphase gerichtet . Auch die Frage, ob Prekarität subjektiv so erlebt wird oder nicht, ist noch nicht ausreichend erforscht.

In dieser Studie zeigen wir auf, wie bestehende Forschung in mehrfacher Hinsicht erweitert werden kann . Dazu betrachten wir im Unterschied zu vielen anderen Studien die letzte Erwerbsphase von Männern und Frauen und analysieren auf Basis von Individual- und Paardaten des Sozioökonomischen Panels (2008-2014) inwieweit a) unterschiedliche Dimensionen und Grade von Prekarität, b) die Kumulation von Prekarität in der Beschäftigungshistorie, sowie c) die Kumulation von Prekarität in Partnerschaften die subjektive Prekaritätswahrnehmung steigern . Die Ergebnisse unserer Analysen zeigen, dass vier Prekaritätsdimensionen - Beschäftigungsprekarität, Einkommensprekarität, Prekarität der Beschäftigungsfähigkeit und sozialrechtliche Prekarität - einen signifikanten Einfluss auf die subjektive Wahrnehmung der eigenen Arbeitsplatzsicherheit haben und diese wechselseitig verstärken. Gleichzeitig steigert die Kumulation von Prekarität in der Beschäftigungshistorie sowie die Kumulation von Prekarität in der Partnerschaft die subjektive Prekaritätswahrnehmung . 


\section{Subjektive Erwerbsprekarität als sozial bedingter kognitiver Evaluationsprozess}

Nationale und internationale Studien haben die zunehmende Bedeutung von prekären Beschäftigungsverhältnissen und ihren Folgen z.B . für den Erwerbsverlauf, Gesundheit, das individuelle Wohlergehen, die Partnerschaft, oder Fertilität vielfach gezeigt (z.B. Allmendinger et al . 2013; Buchholz et al . 2009; Golsch 2005). Dabei wird deutlich, dass Erwerbsprekarität - gekennzeichnet durch geringen Schutz vor Arbeitsplatzverlust, nicht existenzsichernde Einkommen, begrenzten Zugang zu

beruflich-betrieblicher Weiterbildung und/oder eingeschränkte sozialrechtliche

Ansprüche - gesellschaftliche Teilhabebedingungen und -chancen von jungen Erwachsenen einschränkt . Vor dem Hintergrund bedeutsamer Veränderungen der Rahmenbedingungen für Beschäftigung, Verrentung und soziale Sicherung seit den 1990er Jahren ist aber gleichzeitig davon auszugehen, dass viele Prekaritätsrisiken auch in der späteren Erwerbsphase zum Tragen kommen - insbesondere dann, wenn sich dieFolgen prekärerBeschäftigungenüberden individuellenErwerbsverlaufund auch über verschiedene Lebensbereiche kumulieren konnten . Eine Frage, die sich hieran anschließt, ist, ob diese Prekarität auch subjektiv so erlebt wird oder nicht.

Bisher ist zur subjektiven Wahrnehmung von Erwerbsprekarität wenig bekannt, insbesondere für Deutschland (Chung und Mau 2014; Hense i.E.). Der Fokus auf subjektive Erwerbsprekarität ist jedoch von wissenschaftlichem und gesellschaftlichem Interesse, da bereits die Antizipation eines Erwerbsverlustes entsprechende Folgen zum Beispiel für die Gesundheit aufweisen kann, auch wenn der Erwerbsverlust nicht eintritt (Hense i.E.).

Abb . 1 basiert auf Umfragedaten des Sozio-oekonomischen Panels (SOEP) und unterstreicht, dass subjektive Erwerbsprekarität ein bedeutsames Phänomen in der letzten Erwerbsphase darstellt: $42 \%$ der Männer und 38\% der Frauen sorgen sich um die Sicherheit ihres Arbeitsplatzes.

In der Literatur wird diese subjektiv empfundene Prekarität als Ergebnis eines individuellen Evaluationsprozesses aus der Wahrnehmung der Wahrscheinlichkeit undderBedeutung des Erwerbsverlustes beschrieben(Andersonund Pontusson 2007; Chung und Mau 2014; für einen wegweisenden Artikel: Greenhalgh und Rosenblatt 1984) . Dieser Evaluationsprozess ergibt sich zum einen aus den zur Verfügung stehenden Ressourcen, die die Wahrscheinlichkeit des Erwerbsverlustes und/oder die Konsequenzen eines Erwerbsverlustes bedingen (Chung und Mau 2014; Hense i.E.). Bestehende Forschung hat hier gezeigt, dass die Arbeitsmarktbedingungen (z.B. hohe Arbeitslosigkeit), das Beschäftigungsverhältnis (z.B. Befristung), die individuelle Beschäftigungsfähigkeit (z. B . Humankapital), die Haushaltssituation (z.B. Haushaltseinkommen) und sozialrechtliche Maßnahmen (z. B. aktivierende 


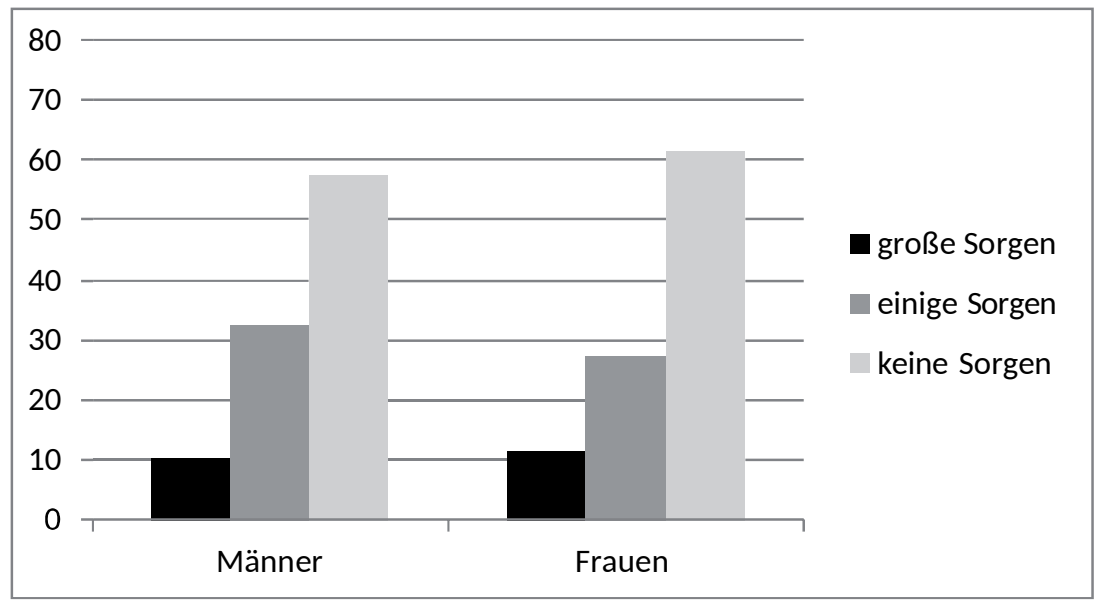

Abb. 1 Sorgen um die Sicherheit des Arbeitsplatzes in der späten Erwerbsphase (in \%) Anmerkung: Erwerbstätige Männer und Frauen im Alter von 50-65 Jahren (ohne Selbstständige) .

Datenbasis: SOEP v.31.1, 2014, eigene Berechnungen.

Arbeitsmarktpolitiken, soziale Sicherungspolitiken) Einfluss auf das subjektive Prekaritätsempfinden nehmen (für einen Überblick siehe Anderson und Pontusson 2007; Chung und Mau 2014; Hense i.E.).

Zum anderen wird der Evaluationsprozess der eigenen Erwerbsprekarität von bestehenden Erfahrungen mit Unsicherheiten und den eigenen Möglichkeiten damit umzugehen beeinflusst, da diese das Wahrnehmungsschemata prägen (Chung und Mau 2014; Hense i.E.). Auch Gefhen et al. (2015, S . 118) zeigen in ihrer qualitativen Studie, dass Personen in prekären Beschäftigungsverhältnissen diese unterschiedlich interpretieren und bewerten, da sich die subjektive Deutung von Erwerbsprekarität ,in ständiger Wechselwirkung zwischen den (erwerbsbiografisch häufig wechselnden) Arbeitsbedingungen und den zu ihrer Deutung jeweils herangezogenen Vorstellungen von Normalität" vollzieht.

Wir möchten im Rahmen dieses Forschungsbeitrages die Daten des Sozio-oekonomischen Panels (2008-2014) nutzen, um drei Forschungsfragen zu untersuchen: Erstens betrachten wir Prekarität in der späten Erwerbsphase multidimensional und fragen, inwieweit sich subjektive Erwerbsprekarität aus der Kumulation von verschiedenen Prekaritätsdimensionen im Beschäftigungsverhältnis erklären lässt . Ausgehend von der Lebensverlaufsforschung (Mayer 2006), die auf den Lebenslauf 
als selbstreferentiellen Prozess mit Pfadabhängigkeiten und die Interdependenz von Lebensläufen von Partnern verweist, sind zwei weitere Fragen bedeutsam . Zum einen untersuchen wir, welche Bedeutung der Kumulation von Prekarität in der Beschäftigungshistorie zukommt. Zum anderen widmen wir uns der Frage, inwiefern auch die Kumulation von Prekarität in der Partnerschaft eine Rolle für die Prekaritätswahrnehmung spielt . Dieser Fokus impliziert eine Betrachtung von Prekarität in ihren vielfältigen Facetten und in ihrem unterschiedlichen Ausmaß . Unser Beitrag gliedert sich wie folgt: Der nächste Abschnitt gibt einen Überblick über den theoretischen Rahmen und Forschungsstand. Im Anschluss beschreiben wir die verwendeten Daten sowie unser methodisches Vorgehen . Danach stellen wir die empirischen Befunde unserer Analyse vor . Der Beitrag schließt mit einer Zusammenfassung und einem Fazit .

\section{Implikationen der Kumulation von Prekaritätfür subjektive Erwerbsprekarität}

\subsection{Prekaritätsdimensionen und ihre Kumulation}

Prekarität ist ein multidimensionales Konstrukt, das jedoch in quantitativen Studien bislang kaum multidimensional und im Längsschnitt betrachtet wurde (Brehmer und Seifert 2008). Am häufigsten wird Erwerbsprekarität als Gefährdung der erwerbsbezogenen Teilhabe sowie Einkommensprekarität untersucht . Jedoch findet man in der Literatur auch andere Dimensionen der Prekarität wie z. B. sozialrechtliche Prekarität oder Prekarität der Beschäftigungsfähigkeit.Im Folgenden argumentieren wir, dass das Ausmaß der Prekarität von Beschäftigungsverhältnissen anhand von vier Dimensionen erhoben werden kann, die jeweils auch die subjektive Wahrnehmung der Wahrscheinlichkeit und der Bedeutung des Erwerbsverlustes bedingen .

Beschäftigungsprekarität zeichnet sich durch einen geringen Schutz vor dem Verlust des Arbeitsplatzes aus. Dies gilt insbesondere für befristete Beschäftigungsverhältnisse, aber auch für Zeit- und Leiharbeit oder Betriebe, die vermehrt Kündigungen aussprechen, z . B . aus ökonomischen Gründen . Damit geht eine

höhere Wahrscheinlichkeit eines Erwerbsverlustes einher, die das subjektive Prekaritätsempfinden erhöht . Dementsprechend hat auch bestehende Forschung gezeigt, dass bei befristet Beschäftigten (Erlinghagen 2008; Mau et al .2012) sowie Beschäftigten, die die ökonomische Situation ihres Betriebes negativ beurteilen, subjektive Erwerbsprekarität besonders hoch ist (Mauno und Kinnunen 2002). Einkommensprekariät adressiert Erwerbseinkommen, die nicht existenzsichernd 
sind . Ein Niedriglohneinkommen impliziert, dass ein potentieller Erwerbsverlust besonders bedeutsam ist, da er nicht durch angespartes Einkommen aufgefangen werden kann (Brehmer und Seifert 2008; Keller und Seifert 2011). Prekarität der Beschäftigungsfähigkeit bezeichnet eine geringe Akkumulation von Humankapital im derzeitigen Erwerbsverhältnis, die es schwierig macht, bei Verlust des Arbeitsplatzes schnell einen neuen Arbeitsplatz zu finden und neuen Qualifikationsanforderungen nachzukommen (Brehmer und Seifert 2008; Keller und Seifert 2011). Dies gilt zum Beispiel für Tätigkeiten ohne Zugang zur beruflich-betrieblichen Weiterbildung, Tätigkeiten, die keine Ausbildung erfordern, oder berufliche Tätigkeiten, die nicht dem gelernten Beruf entsprechen . Ein potentieller Erwerbsverlust wird in diesem Rahmen besonders negativ bewertet, da die Suche nach einem neuen Arbeitsverhältnis aufgrund einer niedrigen Akkumulation von Humankapital oder einer Entwertung von Humankapital schwer erscheint. Sozialrechtliche Prekarität thematisiert schließlich eine fehlende Absicherung von Erwerbsverlust durch bestehendes Recht sowie eine geringe sozialrechtliche Absicherung vor finanziellen Notlagen durch Erwerbsverlust (Brehmer und Seifert 2008; Keller und Seifert 2011). Ersteres gilt für Beschäftigte in Betrieben mit geringer Beschäftigungszahl, für die bestimmte rechtliche Kündigungsschutzregelungen nicht gelten . Letzteres gilt für Beschäftigte, die in den letzten zwei Jahren nicht erwerbstätig waren, und somit kein Anrecht auf Sozialleistungen genießen . Somit sind größere Sorgen um die Sicherheit des Arbeitsplatzes wahrscheinlich, da rechtliche Ressourcen zum Schutz vor Erwerbsverlust und zum Schutz vor negativen Konsequenzen des Erwerbsverlustes fehlen. In der Tat zeigen Studien für Deutschland, dass mit zunehmender Unternehmensgröße eine Reduktion der Prekaritätswahrnehmung einhergeht (Erlinghagen 2008).

Auf Basis der vorangegangenen Erläuterungen formulieren wir folgende Hypothese 1: Bei Beschäftigten mit Beschäftigungsverhältnissen, die sich durch hohe Beschäftigungsprekarität, Einkommensprekarität, Prekarität der Beschäftigungsfähigkeit und/oder sozialrechtliche Prekarität auszeichnen, sind Sorgen um die Sicherheit des Arbeitsplatzes besonders hoch.

In Anlehnung an das Konzept der Kumulation von Vor- und Nachteilen (DiPrete und Eirich 2006), das unter anderem darauf verweist, dass Risiken oder negative Erfahrungen das Erleben von anderen Risiken oder negativen Erfahrungen wahrscheinlicher machen, gehen wir des Weiteren davon aus, dass sich die verschiedenen Prekaritätsdimensionen in ihrem Einfluss auf subjektive Erwerbsprekarität verstärken . Treten mehrere Prekaritätsdimensionen gemeinsam auf, bedeutet dies, dass Prekaritätserfahrungen nicht durch andere Ressourcen abgemildert werden können . Ein Erwerbsverlust sowie entsprechende negative Konsequenzen sind zum Beispiel besonders wahrscheinlich, wenn Beschäftigungsprekarität mit 
Einkommensprekarität, Prekarität der Beschäftigungsfähigkeit oder sozialrechtlicher Prekarität einhergeht . Das gleichzeitige Auftreten von mehreren Prekaritätsrisiken sollte, so unsere Vermutung, daher auch die subjektiv wahrgenommene Erwerbsprekarität verschärfen . Zudem wird eine Umdeutung oder Relativierung von prekären Erwerbslagen, wie von Gefhen et al . (2015) beschrieben, erschwert: Beschäftigte in Erwerbssituationen, die in mehrfacher Hinsicht als prekär gelten, nehmen ihre Kompensationsmöglichkeiten für das Nichtvorhandensein eines Normalarbeitsverhältnisses für das eigene Wohlbefinden (z. B . durch soziale Anerkennung, Einbettung in ein soziales Netzwerk, spannende Tätigkeit, Freizeitaktivitäten) als schwerer realisierbar und daher auch die Bedeutung eines möglichen Erwerbsverlustes als besonders hoch wahr. Wir formulieren darauf aufbauend folgende Hypothese 2: Beschäftigungsprekarität, Einkommensprekarität, Prekarität der Beschäftigungsfähigkeit und sozialrechtliche Prekarität verstärken sich in ihrer Relevanz für Sorgen um die Sicherheit des Arbeitsplatzes.

\subsection{Kumulation von Prekarität in der Beschäftigungshistorie}

Auf Basis des Argumentes, dass das Ausmaß der subjektiv empfundenen Erwerbsprekarität von bestehenden Erfahrungen mit Unsicherheiten und den Möglichkeiten damit umzugehen bedingt wird (Chung und Mau 2014; Hense i.E.), gehen wir davon aus, dass vorangegangene Arbeitslosigkeits- und Prekaritätserfahrungen das subjektive Prekaritätsempfinden verstärken . Ein hohes Ausmaß an Arbeitslosigkeitserfahrungen hat Einfluss auf die Ressourcen, die die negativen Implikationen eines potentiellen Erwerbsverlustes abfedern können . Beschäftigte mit langen Arbeitslosigkeitserfahrungen besitzen nur ein geringes $\mathrm{Ma} ß$ an Humankapital in Form von Berufserfahrung. Dies macht einen erneuten Erwerbsverlust wahrscheinlicher. Gleichzeitig wird die Suche nach einem neuen Beschäftigungsverhältnis nach erneutem Erwerbsverlust erschwert, da Erwerbsverläufe, die durch häufige Arbeitslosigkeitsphasen gekennzeichnet sind, aufdem Arbeitsmarkt wenig geschätzt werden. In der Tatzeigen bisherige Studien, dass vergangene Arbeitslosigkeiterneute Arbeitslosigkeit wahrscheinlicher macht (für einen Überblick DiPrete und Eirich 2006) . Arbeitslosigkeitserfahrungen bedeuten aber auch, dass die verschiedenen Implikationen eines Erwerbsverlustes bereits erlebt wurden und so eine Umdeutung und Relativierung von Prekarität schwerer scheint . Entsprechend konnten andere Studien bereits Belege dafür finden, dass Erfahrungen von Arbeitslosigkeit die subjektive Prekaritätswahrnehmung verstärken (Erlinghagen 2008; Mau et al . 2012) . Erwerbsprekarität in der Vergangenheit kann in ähnlicher Weise bedeuten, dass bestehende Sorgen durch vorangegangene Sorgen und Erfahrungen verstärkt 
werden, da die Implikationen von Prekarität bereits durchlebt wurden . Daraus lässt sich folgende Hypothese 3 ableiten: Bei Beschäftigten mit vorangegangenen Arbeitslosigkeitserfahrungen und Prekaritätserfahrungen sind Sorgen um die Sicherheit des Arbeitsplatzes besonders hoch.

\subsection{Kumulation von Prekarität in Partnerschaften}

Bisherige Forschung hat gezeigt, dass sich Ressourcen und Belastungen häufig auch in Partnerschaften kumulieren (Blossfeld und Buchholz 2009; Blossfeld und Timm 2003) . Dies gilt auch für prekäre Beschäftigungsverhältnisse (Lange et al. 2013) . Das eröffnet die Frage, welchen Einfluss die Kumulation von Erwerbsprekarität in Partnerschaften für das subjektive Prekaritätsempfinden hat . In der Prekaritätsforschung wird auf die Substitutionsmöglichkeit der Erwerbsarbeit durch den Partner verwiesen (Chung und Mau 2014; Hense i E.). Empirische Ergebnisse belegen, dass mit höherem Haushaltseinkommen die Beschäftigungssorgen sinken (Erlinghagen 2008; Mau et al . 2012). Dabei ist zu berücksichtigen, dass die Erwerbsprekarität des einen Partners je nach Erwerbskonstellation innerhalb einer Partnerschaft zu einer prekären Haushaltslage führen kann, jedoch nicht muss (Andreß und Seeck 2007; Bartelheimer 2011) . Bislang liegen jedoch nur wenige Studien zu „crossover" Prozessen in Partnerschaften vor, mit disparaten Ergebnissen (Mauno und Kinnunen 2002).

Theorien zu Prozessen von „cross-over“ in Partnerschaften spezifizieren drei Mechanismen wie Arbeitsplatzbedingungen des einen Partners einen Einfluss auf das Wohlbefinden des anderen Partners haben (Bakker et al . 2009; Demerouti 2012; Westman 2001) . Zum einen können Stressoren beide Partner beeinflussen . Dies gilt insbesondere für prekäre Beschäftigungsverhältnisse, da ein Erwerbsverlust Einfluss auf die gesamte finanzielle Situation des Haushalts hat . Kumulieren prekäre Beschäftigungsverhältnisse ist der eigene potentielle Erwerbsverlust von noch größerer subjektiver Bedeutung, da ein Erwerbsverlust des Partners ebenfalls als wahrscheinlich gilt . Zweitens ist ,,cross-over" indirekt möglich durch Implikationen der Arbeitsbedingungen des einen Partners für Interaktionen zwischen den Partnern . Belastet das prekäre Beschäftigungsverhältnis die Beziehung mit dem Partner z. B . in dem es zu Konflikten zwischen den Partner führt, hat ein möglicher Erwerbsverlust eine noch größere Relevanz, da sich auch Implikationen für die Partnerschaftsbeziehung ergeben. Kumulieren prekäre Beschäftigungsverhältnisse kann angenommen werden, dass die Partnerschaftsbeziehung durch die Sorgen, die sich beide Partner machen, doppelt belastet wird . Drittens ist ,cross-over“" möglich durch Gefühle wie Empathie für die Situation des anderen Partners. Dieser Mechanismus 
beinhaltet jedoch keine direkten Implikationen für die Kumulation von Prekarität in Partnerschaften. Auf Basis der vorangegangenen Argumente, formulieren wir folgende Hypothese 4: Wenn beide Partner in prekärer Beschäftigung arbeiten, ist die subjektive Prekaritätswahrnehmung besonders hoch.

\section{$3 \quad$ Daten und Methoden}

Als Datengrundlage nutzen wir das Sozio-oekonomische Panel (SOEP v31 1), das es uns ermöglicht, Individual- und Paardaten im Längsschnitt aufzubereiten, um so über die Verknüpfung zeitübergreifender Information unsere zentralen Variablen zu generieren. Konkret betrachten wir Männer und Frauen im Alter von 50-65 Jahren, die zwischen 2008 und 2014 zu mindestens einem Erhebungszeitpunkt abhängig beschäftigt waren und in einer Partnerschaft lebten . Die abhängige Variable in den Analysen ist die Frage nach den Sorgen um die Sicherheit des Arbeitsplatzes, eine ordinal skalierte Variable (keine, einige, große Sorgen) . Als Analyseverfahren wählen wir ein RE generalisiertes ordinales Probit Modell (Pfarr et al . 2011). Dieses Analyseverfahren bietet mehrere Vorteile . In den Modellen wird die Wahrscheinlichkeit, sich überhaupt Sorgen zu machen, und die Intensität der Sorgen simultan geschätzt . Dabei wird im Unterschied zu einfachen Probitmodellen die Annahme der parallelen Regression (resp . Proportional-Odds Annahme) aufgegeben (Long 1997). Die einzelnen Einflussfaktoren können somit einen unterschiedlich starken oder auch unterschiedlich gerichteten Einfluss auf die Intensität der Sorgen aufweisen. Lässt man auf diese Weise unterschiedliche Schwellenwerte zu, berücksichtigt man beobachtbare Heterogenität in den Daten . In Stata kann zur Schätzung dieser Modelle der Befehl regoprob2 implementiert werden (Pfarr et al . 2010) . Mithilfe des Wald-Tests wird für jeden der Regressionskoeffizienten die Annahme paralleler Regressionen zunächst geprüft; mehrere Regressionskoeffizienten werden nur bei Verletzung dieser Annahme geschätzt . Als Untersuchungseinheiten gehen die individuellen Befragungspersonen in unsere Modelle ein, für die Partnerinformationen vorliegen . Da die Erwerbstätigkeit von Männern und Frauen sowie die daran geknüpfte Prekarität unterschiedliche Facetten aufweisen können (Keller et al . 2012; Klammer et al . 2012), schätzen wir getrennte Modelle für Männer und Frauen.

Die zentralen erklärenden Variablen sind zeitveränderliche Dummyvariablen zur Beschreibung der unterschiedlichen Dimensionen und Grade von Erwerbsprekarität im derzeitigen Beschäftigungsverhältnis, der Prekaritätserfahrung sowie der Kumulation von Prekarität in der Partnerschaft . Tab . 1 zeigt für jede der vier 
in der Literatur diskutierten Prekaritätsdimensionen die verwendeten Indikatoren aus dem SOEP . Die Kumulation dieser Prekaritätsdimensionen bilden wir anhand von vier Dummyvariablen ab (auf keiner, einer, zwei, drei und vier Dimensionen prekär) . Zwei weitere Dummyvariablen fangen die Kumulation von Prekarität in der Beschäftigungshistorie ein: die Arbeitslosigkeitserfahrung in den letzten fünf Jahren sowie vorangegangene Phasen der Erwerbsprekarität in den letzten drei Jahren. In den Partnermodellen berücksichtigen wir nur noch Partnerschaften, in denen beide Partner erwerbstätig sind . Unser Augenmerk liegt dann auf vier Dummyvariablen, die für jede der vier Prekaritätsdimensionen jeweils anzeigen, ob beide Partner prekär sind. Als Kontrollvariablen berücksichtigen wir in allen Modellen das Alter, das Bildungsniveau (niedrig, mittel, hoch), die selbsteingeschätzte Chance, eine andere geeignete Stelle zu finden (möglich, unmöglich), die Migrationserfahrung (keine, direkte, indirekte), die Wohnregion (alte, neue Bundesländer) sowie die Untersuchungsjahre (2008-2014). Auch nach Kontrolle dieser Variablen ist denkbar, dass personenspezifische Eigenschaften der Untersuchungspersonen unberücksichtigt bleiben, die sowohl den Grad der Erwerbsprekarität als auch die Wahrscheinlichkeit, sich Sorgen zu machen, beeinflussen . Abschließend sei an dieser Stelle darauf hingewiesen, dass wir die Panelstruktur nicht nutzen, um diese verbleibende unbeobachtete Heterogenität zu kontrollieren.

Tab. 1 Prekaritätsdimensionen und verwendete Indikatoren

\begin{tabular}{ll}
\hline Prekaritätsdimension & Indikatoren im SOEP \\
\hline Beschäftigung & $\bullet$ Befristung, Zeit- und Leiharbeit \\
& - Verringerung der Belegschaft in den letzten 12 Monaten \\
Einkommen & - Niedriglohn $(<2 / 3$ des Medianeinkommens $)$ \\
Beschäftigungsfähigkeit & • Tätigkeit, für die keine Ausbildung erforderlich ist \\
& - Tätigkeit nicht im erlernten Beruf \\
Sozialrechtlich & - Tätigkeit in einem Betrieb ohne Kündigungsschutz \\
& - Keine Anrechte auf Sozialleistungen \\
\hline
\end{tabular}




\section{$4 \quad$ Ergebnisse}

Tab. 2 Einflussfaktoren auf Sorgen um den Arbeitsplatz (Individualmodell)

\begin{tabular}{|c|c|c|c|c|c|c|c|}
\hline \multirow{3}{*}{ Prekarität aktueller Job (Ref . Nein) } & \multirow[t]{2}{*}{ Sorgen } & \multicolumn{3}{|c|}{ Männer } & \multicolumn{3}{|c|}{ Frauen } \\
\hline & & \multicolumn{2}{|l|}{ Koef. } & \multirow[t]{2}{*}{ Std .Err. } & \multicolumn{2}{|l|}{ Koef. } & \multirow[t]{2}{*}{ Std Err } \\
\hline & & & & & & & \\
\hline & $\begin{array}{l}\mathrm{Ob} \\
\text { Große }\end{array}$ & 0,368 & $* * *$ & $(0,051)$ & $\begin{array}{l}0,466 \\
0,662\end{array}$ & $\begin{array}{l}* * * \\
* * *\end{array}$ & $\begin{array}{l}(0,064) \\
(0,078)\end{array}$ \\
\hline Einkommen & $\begin{array}{l}\mathrm{Ob} \\
\text { Große }\end{array}$ & 0,329 & $* * *$ & $(0,094)$ & 0,231 & $* * *$ & $(0,071)$ \\
\hline Beschäftigungsfähigkeit & $\begin{array}{l}\mathrm{Ob} \\
\text { Große }\end{array}$ & 0,179 & $* * *$ & $(0,061)$ & 0,043 & & $(0,071)$ \\
\hline Sozialrechtlich & $\begin{array}{l}\mathrm{Ob} \\
\text { Große }\end{array}$ & 0,334 & $* * *$ & $(0,097)$ & 0,100 & & $(0,078)$ \\
\hline Prekaritätserfahrung (Ref . Nein) & & & & & & & \\
\hline Arbeitslosigkeit in letzten 5 Jahren & $\begin{array}{l}\mathrm{Ob} \\
\text { Große }\end{array}$ & 0,483 & $* * *$ & $(0,082)$ & 0,390 & $* * *$ & $(0,085)$ \\
\hline Prekarität in letzten 3 Jahren & $\begin{array}{l}\mathrm{Ob} \\
\text { Große }\end{array}$ & 0,060 & & $(0,055)$ & $\begin{array}{r}0,186 \\
-0,081 \\
\end{array}$ & $* * *$ & $\begin{array}{l}(0,066) \\
(0,085)\end{array}$ \\
\hline Alter & $\begin{array}{l}\mathrm{Ob} \\
\text { Große }\end{array}$ & 1,269 & $* * *$ & $(0,172)$ & $\begin{array}{l}0,787 \\
0,827\end{array}$ & $\begin{array}{l}* * * \\
* * *\end{array}$ & $\begin{array}{l}(0,204) \\
(0,203)\end{array}$ \\
\hline Alter zum Quadrat & $\begin{array}{l}\mathrm{Ob} \\
\text { Große }\end{array}$ & $-0,012$ & $* * *$ & $(0,002)$ & $-0,008$ & $* * *$ & $(0,002)$ \\
\hline Bildung (Ref .: Hoch) & & & & & & & \\
\hline Niedrig & $\begin{array}{l}\mathrm{Ob} \\
\text { Große }\end{array}$ & $-0,126$ & & $(0,162)$ & 0,585 & $* * *$ & $(0,155)$ \\
\hline Mittel & $\begin{array}{l}\mathrm{Ob} \\
\text { Große }\end{array}$ & 0,435 & $* * *$ & $(0,081)$ & $\begin{array}{l}0,296 \\
0,549\end{array}$ & $\begin{array}{l}* * * \\
* * *\end{array}$ & $\begin{array}{l}(0,099) \\
(0,112)\end{array}$ \\
\hline Chance Stelle zu finden (Ref. Ja) & & & & & & & \\
\hline Nein & $\begin{array}{l}\mathrm{Ob} \\
\text { Große }\end{array}$ & $\begin{array}{l}0,105 \\
0,458\end{array}$ & $\begin{array}{l}* \\
* * *\end{array}$ & $\begin{array}{l}(0,060) \\
(0,070)\end{array}$ & $\begin{array}{l}0,168 \\
0,395\end{array}$ & $\begin{array}{l}* * \\
* * *\end{array}$ & $\begin{array}{l}(0,065) \\
(0,079)\end{array}$ \\
\hline Migrationserfahrung (Ref .: Keine) & & & & & & & \\
\hline Direkte & $\begin{array}{l}\mathrm{Ob} \\
\text { Große }\end{array}$ & 0,982 & $* * *$ & $(0,121)$ & 0,909 & $* * *$ & $(0,153)$ \\
\hline Indirekte & $\begin{array}{l}\mathrm{Ob} \\
\text { Große }\end{array}$ & 0,244 & $*$ & $(0,144)$ & $-0,133$ & & $(0,180)$ \\
\hline Wohnregion (Ref .: Alte Bundeslän & & & & & & & \\
\hline Neue Bundesländer & $\begin{array}{l}\mathrm{Ob} \\
\text { Große }\end{array}$ & $\begin{array}{l}0,888 \\
0,548\end{array}$ & $\begin{array}{l}* * * \\
* * *\end{array}$ & $\begin{array}{l}(0,094) \\
(0,103)\end{array}$ & $\begin{array}{l}1,007 \\
0,778\end{array}$ & $\begin{array}{l}* * * \\
* * *\end{array}$ & $\begin{array}{l}(0,100) \\
(0,110)\end{array}$ \\
\hline
\end{tabular}




\begin{tabular}{|c|c|c|c|c|c|c|c|}
\hline \multicolumn{8}{|l|}{ Jahr (Ref .: 2008) } \\
\hline 2009 & $\begin{array}{l}\mathrm{Ob} \\
\text { Große }\end{array}$ & 0,218 & $* * *$ & $(0,067)$ & 0,156 & $* *$ & $(0,074)$ \\
\hline 2010 & $\begin{array}{l}\mathrm{Ob} \\
\text { Große }\end{array}$ & 0,183 & $* *$ & $(0,073)$ & 0,179 & $* *$ & $(0,078)$ \\
\hline 2011 & $\begin{array}{l}\mathrm{Ob} \\
\text { Große }\end{array}$ & $-0,361$ & $* * *$ & $(0,075)$ & $-0,235$ & $* * *$ & $(0,082)$ \\
\hline 2012 & $\begin{array}{l}\mathrm{Ob} \\
\text { Große }\end{array}$ & $-0,099$ & & $(0,076)$ & 0,100 & & $(0,083)$ \\
\hline 2013 & $\begin{array}{l}\mathrm{Ob} \\
\text { Große }\end{array}$ & $-0,230$ & $* * *$ & $(0,076)$ & $-0,073$ & & $(0,083)$ \\
\hline 2014 & $\begin{array}{l}\mathrm{Ob} \\
\text { Große }\end{array}$ & $-0,318$ & $* * *$ & $(0,078)$ & $\begin{array}{r}-0,187 \\
0,044\end{array}$ & $* *$ & $\begin{array}{l}(0,091) \\
(0,111)\end{array}$ \\
\hline Konstante & $\begin{array}{l}\mathrm{Ob} \\
\text { Große }\end{array}$ & $\begin{array}{l}-33,708 \\
-37,396\end{array}$ & $\begin{array}{l}* * * \\
* * *\end{array}$ & $\begin{array}{l}(4,812) \\
(4,793)\end{array}$ & $\begin{array}{l}-20,440 \\
-24,809\end{array}$ & $\begin{array}{l}* * * \\
* * *\end{array}$ & $\begin{array}{l}(5,695) \\
(5,650)\end{array}$ \\
\hline $\begin{array}{l}\text { Log likelihood } \\
\text { Wald } \chi^{2}(23)\end{array}$ & & $\begin{array}{r}-4873 \\
697,22\end{array}$ & $* * *$ & & $\begin{array}{r}-4231 \\
492,63\end{array}$ & $* * *$ & \\
\hline $\begin{array}{l}\rho \\
\mathrm{N}\end{array}$ & & $\begin{array}{r}0,672 \\
6534\end{array}$ & $* * *$ & & $\begin{array}{r}0,664 \\
5961\end{array}$ & $* * *$ & \\
\hline
\end{tabular}

Anmerkungen: Random effects generalized ordered probit Modell, Standardfehler in Klammern. ${ }^{*} \mathrm{p}<.10 ;{ }^{* *} \mathrm{p}<.05 ;{ }^{* * *} \mathrm{p}<.01$.

Datenbasis: SOEP v.31.1, 2008-2014, eigene Berechnungen.

Wie in Hypothese 1 vermutet geht Erwerbsprekarität mit einer höheren Wahrscheinlichkeit einher, sich Sorgen um den Arbeitsplatz zu machen . Tab . 2 dokumentiert, dass bei Männern jede der vier Prekaritätsdimensionen signifikant die Sorgen um die Sicherheit des Arbeitsplatzes sowie die Intensität der Sorgen beeinflusst . Demgegenüber zeigen bei Frauen nur zwei Dimensionen einen signifikanten Einfluss: die Beschäftigungsprekarität und die Einkommensprekarität, wobei Ersteres die Intensität der Sorgen von Frauen noch zusätzlich steigert . Dieser unterschiedliche Einfluss der vier Prekaritätsdimensionen für Männer und Frauen steht vermutlich damit im Zusammenhang, dass viele Paare insbesondere in den alten Bundesländern nach dem Hinzuverdiener-Modell leben, bei dem der Mann Vollzeit und die Frau Teilzeit arbeitet . In diesem Modell der Arbeitsteilung hat gerade eine prekäre Erwerbslage des Mannes weitreichende Konsequenzen für die ökonomische Absicherung einer Familie . Demgegenüber spielt vermutlich eine geringe Akkumulation von Humankapital oder etwa eine fehlende Absicherung von Erwerbsverlust durch bestehendes Recht eine geringere Rolle für die Frau 
als Hinzuverdienerin im Haushalt . Da die in Tab . 2 betrachteten Modelle jedoch ausschließlich auf Individualdaten - und nicht auf Paardaten - fußen, lassen sich diese Überlegungen noch nicht empirisch abstützen .

Wird die Prekarität von Beschäftigungsverhältnissen mehrdimensional gemessen, so ist es auch möglich, die Intensität von Prekarität abzubilden. Entsprechend betrachten wir in Tab . 3 die kumulative Wirkung der Prekaritätsdimensionen . Im Vergleich zu Männern und Frauen, deren aktueller Job nicht prekär ist, steigt die Wahrscheinlichkeit sich Sorgen um den Arbeitsplatz zu machen je mehr Prekaritätsdimensionen den aktuellen Job einer Person kennzeichnen . Unterschiedliche Grade von Prekarität werden demnach auch subjektiv unterschiedlich erlebt - ein Befund, der unsere Hypothese 2 erhärtet.

Tab. 3 Einflussfaktoren auf Sorgen um den Arbeitsplatz (Individualmodell)

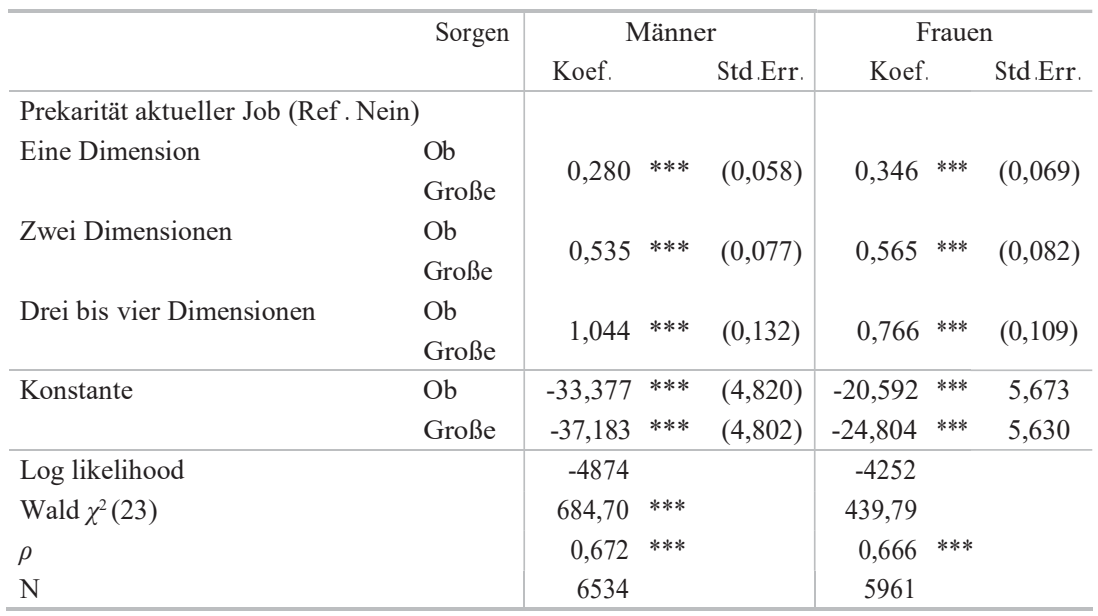

Anmerkungen: Auszug aus dem Schätzmodell, das auch die Prekaritätserfahrung und alle Kontrollvariablen beinhaltet . Random effects generalized ordered probit Modell, Standardfehler in Klammern. ${ }^{*} \mathrm{p}<.10 ;{ }^{* *} \mathrm{p}<.05 ;{ }^{* * *} \mathrm{p}<.01$

Datenbasis: SOEP v.31.1, 2008-2014, eigene Berechnungen.

Hinsichtlich der Prekaritätserfahrung über den Lebenslauf ist festzustellen, dass Männer und Frauen mit Arbeitslosigkeitserfahrungen sich häufiger Sorgen um ihren Arbeitsplatz machen (Tab . 2). Vorangegangene Phasen der Erwerbsprekarität hingegen haben in dem Modell für die Männer keinen signifikanten Einfluss .Bei 
den Frauen erhöht sich die Wahrscheinlichkeit, sich überhaupt Sorgen zu machen, der Grad der Sorgen wird jedoch nicht beeinflusst . Die dritte Hypothese kann daher nur teilweise bestätigt werden . Um dem Einfluss der Prekaritätserfahrung auf den Grund zu gehen, bedarf es noch weiter ausdifferenzierter Analysen . Denkbar ist, dass sich hier ein Gewöhnungseffekt verbirgt und Frauen und Männer, die schon länger Erwerbsprekarität erfahren, sich tatsächlich nicht häufiger Sorgen um ihren Arbeitsplatz machen . Plausibel ist aber auch, dass nicht allein die Prekaritätserfahrung an sich, sondern vielmehr die Intensität der erlebten Prekarität (gemessen anhand der Anzahl der Prekaritätsdimensionen) bedeutsam ist, die wir in den Modellen jedoch nicht berücksichtigen. So ist z . B . zu vermuten, dass Männer Beschäftigungsprekarität dann bewusst in Kauf nehmen, wenn sie eine hohe Entlohnung erhalten . Dagegen ist die Erwerbssituation von Frauen häufiger auf mehreren Dimensionen als prekär zu beschreiben .

Die anderen Einflussfaktoren (Tab . 1) zeichnen ein Bild, das den Befunden der bisherigen Forschung entspricht (Hense i.E.). Hinsichtlich des Alters beobachten wir einen u-förmigen Zusammenhang: Mit steigendem Alter vergrößern sich die Sorgen um den Arbeitsplatz. Je näher das Renteneintrittsalter rückt, desto schwächer wird jedoch dieser Alterseffekt . Bildung beeinflusst nicht nur die Wahrscheinlichkeit eines Erwerbsverlustes, sondern auch die Chancen, eine neue Beschäftigung zu finden . Entsprechend zeigen unsere Analysen, dass Männer und Frauen mit einer hohen Bildung sich seltener Sorgen um ihren Arbeitsplatz machen . Beschreiben sie ihre Chancen, eine neue Stelle zu finden, als aussichtslos, erhöht dies die Sorgen um die Arbeitsplatzsicherheit sowie die Intensität der Sorgen . Die Wahrnehmung der Erwerbsprekarität ist auch stärker ausgeprägt bei Personen mit direkter Migrationserfahrung. Männer und Frauen aus den neuen Bundesländern machen sich ebenfalls häufiger Sorgen um ihren Arbeitsplatz . Die Dummies für die einzelnen Untersuchungsjahre spiegeln vermutlich die Folgen der Finanzmarktkrise wider . Die Effekte all dieser Kontrollvariablen sind robust und zeigen sich auch in unseren anderen Modellspezifikationen.

Um die Kumulation von Prekarität in der Partnerschaft zu analysieren, berücksichtigen wir im Folgenden nur noch Partnerschaften, in denen beide Partner erwerbstätig sind. Zuvor ist hervorzuheben, dass sich unsere zentralen Ergebnisse zur Kumulation der unterschiedlichen Prekaritätsdimensionen sowie zur Kumulation von Prekarität in der Beschäftigungshistorie auch bei der Betrachtung dieses Analysesamples zeigen. Tab . 4 stellt daher nur noch ausgewählte Ergebnisse aus unserem Partnermodell dar. Erneut zeigt sich, dass die eigene Erwerbsprekarität die Sorgen von Männern und Frauen um die Sicherheit ihres Arbeitsplatzes erhöht. Die anderen Variablen in Tab . 4 geben Auskunft über die Kumulation von Prekarität in der Partnerschaft. Für zwei der vier Prekaritätsdimensionen können wir 
einen Beleg für „cross-over“ Prozesse beobachten: Beschäftigungsprekarität und Einkommensprekarität. Wenn sich also nicht nur die Person, sondern gleichzeitig auch der Partner in einer prekären Erwerbslage befindet, die durch einen geringen Schutz vor dem Verlust des Arbeitsplatzes oder Niedriglohn gekennzeichnet ist, so steigert dies das individuelle Prekaritätsempfinden. Wie in Hypothese 4 vermutet, spielt demnach auch der Partnerschaftskontext eine Rolle für die subjektive Wahrnehmung von Erwerbsprekarität, und zwar insbesondere dann, wenn die ökonomische Absicherung einer Familie unsicher ist.

Tab. 4 Einflussfaktoren auf Sorgen um den Arbeitsplatz (Partnermodell)

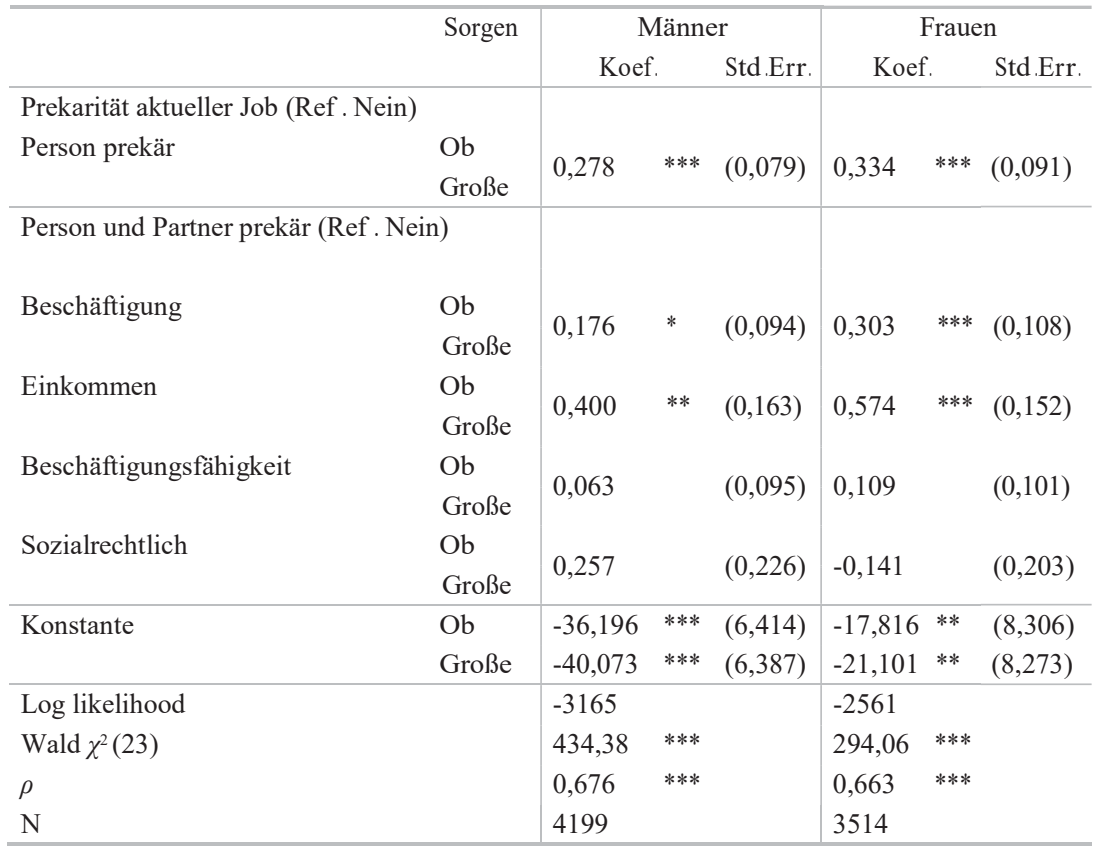

Anmerkungen: Auszug aus dem Schätzmodell, das auch die Prekaritätserfahrung und alle Kontrollvariablen beeinhaltet . Random effects generalized ordered probit Modell, Standardfehler in Klammern. ${ }^{*} \mathrm{p}<.10 ;{ }^{* *} \mathrm{p}<.05 ;{ }^{* * *} \mathrm{p}<.01$.

Datenbasis: SOEP v.31.1, 2008-2014, eigene Berechnungen. 


\section{$4 \quad$ Zusammenfassung und Fazit}

In regelmäßigen Abständen werden neue empirische Studien vorgelegt, die sich mit prekärer Beschäftigung im nationalen und internationalen Vergleich befassen . Diese Studien unterstreichen die zunehmende Bedeutung prekärer Beschäftigung . Dabei wird sehr häufig der Blick auf befristete Beschäftigungsverhältnisse gelenkt und die Gruppe der jungen Erwachsenen als Verlierer bezeichnet. Befristung geht für die einzelnen Akteure oft mit weiteren Nachteilen einher (z. B . niedrigen Verdiensten, größeren Arbeitslosigkeitsrisiken) und führt daher zu einer geringeren Planungssicherheit. In der Folge werden langfristige, bindende Entscheidungen häufig aufgeschoben (Buchholz et al . 2009; Golsch 2005).

In unserem Forschungsbeitrag haben wir aufgezeigt, wie diese Forschung in mehrfacher Hinsicht erweitert werden kann . Erstens ist es wichtig verschiedene Prekaritätsdimensionen in den Blick zu nehmen, um unterschiedliche Grade von Erwerbsprekarität abzubilden. Unsere Analysen zeigen, dass Männer und Frauen sich große Sorgen um die Sicherheit des Arbeitsplatzes machen, wenn sie in Beschäftigungsverhältnissen tätig sind, die sich durch hohe Beschäftigungsprekarität, Einkommensprekarität, Prekarität der Beschäftigungsfähigkeit und/ oder sozialrechtliche Prekarität auszeichnen . Betrachtet man Erwerbsprekarität multidimensional, zeigt sich zudem, dass das gleichzeitige Auftreten von verschiedenen Prekaritätsdimensionen die Sorgen um die Sicherheit des Arbeitsplatzes verstärkt. Im Rahmen zukünftiger Forschung scheint es daher vielversprechend unsere theoretisch begründete Indikatorenauswahl für Erwerbsprekarität zum Beispiel mithilfe von Strukturgleichungsmodellen empirisch zu überprüfen . Eine Erweiterung unseres Ansatzes wäre zudem möglich, wenn auch qualitative Aspekte der Arbeit - wie etwa nicht sinnstiftende Tätigkeit, fehlende soziale Anerkennung, mangelnde soziale Einbindung - empirisch erfasst und damit die Prekarität der Arbeit betrachtet wird (Brinkmann et al . 2006).

Zweitens liefern die vorgestellten Analysen auch einen Beleg dafür, dass die Kumulation von Prekarität in der Beschäftigungshistorie eine Bedeutung für die aktuelle subjektive Prekaritätswahrnehmung hat. Zukünftige Forschung sollte sich daher auf Basis von Längsschnittdaten auch der Verweildauer in Prekaritätsepisoden und speziell der damit einhergehenden subjektiven (Um)deutung von Prekarität widmen .

Drittens sollte in die Betrachtung von Prekarität der Partnerschaftskontext eingebunden werden, da die Folgen prekärer Beschäftigung teilweise durch den Partner gemildert oder aber auch verschärft werden können . Unsere Ergebnisse zeigen, dass die Kumulation von Prekarität in Partnerschaften - konkret die Beschäftigungs- und Einkommensprekarität beider Partner - die Sorgen um die eigene 
Arbeitsplatzsicherheit verstärkt. Somit bietet es sich an, in Zukunft die (un)gleiche Verteilung von Prekarität in Partnerschaften stärker in den Blick zu nehmen, um zu untersuchen, ob weitere Belege für eine zunehmende Ressourcenungleichheit zwischen Partnerschaften gefunden werden können (vgl . dazu auch Blossfeld und Buchholz 2009). In diesem Zusammenhang kann zukünftige Forschung auch noch differenzierter auf unterschiedliche Erwerbsmodelle innerhalb von Partnerschaften im Ost-West-Vergleich eingehen.

Viertens erscheint der Fokus auf Erwerbsprekarität von Männern und Frauen in der letzten Erwerbsphase lohnenswert. Wir haben im Unterschied zu vielen anderen Studien die subjektive Erwerbsprekarität insbesondere von Beschäftigten in der letzten Erwerbsphase betrachtet. Eine weitere Frage die sich hier anschließt ist die nach den langfristigen Folgen von Erwerbsprekarität insbesondere für Alterssicherung und Altersarmut

\section{Literatur}

Allmendinger, J., L. Hipp, und S. Stuth. 2013. Atypical Employment in Europe 1996-2011. Discussion Paper P 2013-003.

Anderson, C., und J. Pontusson. 2007. Workers, worries and welfare states. Social protection and job insecurity in 15 OECD countries. European Journal of Political Research 46:211-235.

Andreß, H.-J, und T. Seeck . 2007. Ist das Normalarbeitsverhältnis noch armutsvermeidend?: Erwerbstätigkeit in Zeiten deregulierter Arbeitsmärkte und des Umbaus sozialer Sicherungssysteme. KZfSS KölnerZeitschrift für Soziologieund Sozialpsychologie 59:459-492.

Bakker, A. B., M. Westman, und I. J. H.v. Emmerik. 2009. Advancements in crossover theory. Journal of Managerial Psychology 24: 206-219.

Bartelheimer, P. 2011. Unsichere Erwerbsbeteiligung und Prekarität. WSI-Mitteilungen: 386-393.

Blossfeld, H.-P., und S. Buchholz. 2009. Increasing Resource Inequality among Families in Modern Societies: The Mechanisms of Growing Educational Homogamy, Changes in the Division of Work in the Family and the Decline of the Male Breadwinner Model. Journal of Comparative Family Studies 40: 603-616.

Blossfeld, Hans-Peter, und A. Timm. 2003. Who marries whom? Dordrecht, Boston, London: Kluwer.

Brehmer, W ., und H . Seifert . 2008 . Sind atypische Beschäftigungsverhältnisse prekär? - eine empirische Analyse sozialer Risiken. Zeitschrift für ArbeitsmarktForschung 41: 501-531 .

Brinkmann, U., K. Dörre, S. Röbenack, K. Kraemer, und F. Speidel. 2006. Prekäre Arbeit. Ursachen, Ausmaß, soziale Folgen und subjektive Verarbeitungsformen unsicherer Beschäftigungsverhältnisse. Bonn: Friedrich-Ebert-Stiftung.

Buchholz, S., D. Hofäcker, M. Mills, H.-P. Blossfeld, K. Kurz, und H. Hofmeister. 2009. Life Courses in the Globalization Process: The Development of Social Inequalities in Modern Societies . European Sociological Review 25: 53-71. 
Chung, H . und S . Mau . 2014 . Subjective insecurity and the role of institutions . Journal of European Social Policy 24: 303-318.

Demerouti, E. 2012. The Spillover and Crossover of Resources Among Partners: The Role of Work-Self and Family-Self Facilitation. Journal of Occupational Health Psychology 17: 184-195.

DiPrete, T. A ., und G. M. Eirich. 2006. Cumulative advantage as a mechanism for inequality: A review of theoretical and empirical developments. Annual Review of Sociology 32: 271-297.

Erlinghagen, M.2008. Self-perceived job insecurity and social context: A multi-level analysis of 17 European countries. European Sociological Review 24: 183-197.

Gefhen, A., F. Stockem, und P. Böhnke. 2015. Subjektive Umgangsformen mit prekärer Erwerbsarbeit-Zwischen Orientierung an und Ablösung von der Normalarbeitsgesellschaft . Berliner Journal für Soziologie 25: 111-131.

Golsch, Katrin. 2005. The impact of labour market insecurity on the work and family life of men and women. Frankfurt am Main [u. a.]: Lang

Greenhalgh, L., und Z. Rosenblatt. 1984. Job insecurity: Towards conceptual clarity. Academy of Management Review 9: 438-448.

Hense, Andrea. i.E. Wahrnehmung der eigenen Prekarität Grundlagen einer Theorie zur sozialen Erklärung von Ungleichheitswahrnehmungen: VS Verlag für Sozialwissenschaften .

Keller, Bernd, und H. Seifert. (2011): Atypische Beschäftigung. Flexibilisierung und soziale Risiken, Berlin: Friedrich-Ebert-Stifung .

Keller, B., S. Schulz, und H. Seifert. 2012. Entwicklungen und Strukturmerkmale der atypisch Beschäftigten in Deutschland bis 2010 . WSI - Diskussionspapier Nr . 182 .

Klammer, U., S. Neukirch, und D. Weßler-Poßberg. 2012. Wenn Mama das Geld verdient: Familienernährerinnen zwischen Prekarität und neuen Rollenbildern . Bd . 139 . Berlin: Edition Sigma.

Lange, M. de, M. H. J. Wolbers, und W. C. Ultee. 2013. United in Precarious Employment? Employment Precarity of Young Couples in the Netherlands, 1992-2007. European Sociological Review 29: 503-516.

Long, J . Scott . 1997 . Regression models for categorical and limited dependent variables . Thousand Oaks, Calif: Sage Publ .

Mau, S., J. Mewes, und N. M. Schöneck. 2012. What determines subjective socioeconomic-insecurity? Context and class in comparative perspective. Socio-Economic Review 10: 665-682 .

Mauno, S., und U. Kinnunen. 2002 . Perceived job insecurity among dual-earner couples: Do its antecedents vary according to gender, economic sector and the measure used? Journal of Occupational and Organizational Psychology 75: 295-314 .

Mayer, K.U. 2009. New directions in life course research. Annual Review of Sociology 35: 413-433.

Pfarr, C., A. Schmid, und U. Schneider. 2010. REGOPROB2: Stata module to estimate random-effects generalized ordered probit models (update), Statistical Software Components, Boston College Departement of Economics .

Pfarr, C., A. Schmid, und U. Schneider. 2011. Estimating Ordered Categorical Variables Using Panel Data: A Generalised Ordered Probit Model with an Autofit Procedure. Journal of Economics and Econometrics 54: 7-23.

Wagner, G., Frick, J., und J. Schupp. 2007. The German Socio-Economic Panel Study (SOEP) - Scope, Evolution and Enhancements. Schmollers Jahrbuch 127(1): 139-169.

Westman, M.2001. Stress and strain crossover. Human Relations 54: 717-751. 